\title{
Détermination du coefficient de frottement du fond pour l'action combinée de la houle et du courant d'écoulement
}

\author{
M.Hassane $^{(\mathrm{a})}$, M.S Benhafid ${ }^{(\mathrm{b})}$ \& A.Ayadi ${ }^{(\mathrm{c})}$ \\ Ecole Nationale Supérieure de l'Hydraulique (ENSH) de Blida, BP 31 \\ Blida (09000)- Algérie \\ $\overline{\mathrm{Tel}}:+213 .(0) 25.39 .94 .47 ; \quad \overline{\mathrm{Fax}}:+213 .(0) 25.39 .94 .46$ \\ (a) hassanemed@yahoo.fr ; (b)mohbenhafid@yahoo.fr ; \\ (c)abdayadi@yahoo.fr
}

\section{Résumé}

Les vagues de vent générées sur la surface libre des courants dans les cours d'eau (fleuves, grands canaux, etc.), les retenues et en zone côtière, sont susceptibles de donner naissance à des vitesses de fond comparables à celles des courants de décharge. A cet effet, il est indispensable de prendre en considération, dans le calcul des érosions générales et locales, l'influence simultanée de la houle et des courants d'écoulement sur le mouvement des sédiments.

La contrainte de cisaillement au fond, proportionnelle au coefficient de frottement au fond, est le principal paramètre de détermination de la dynamique des sédiments. Donc la détermination du coefficient de frottement au fond dans les conditions de l'action combinée de la houle et des courants représente un intérêt scientifique et pratique certain.

Nous avons examiné les différentes méthodes existantes de détermination du coefficient de frottement au fond dans les conditions de l'action combinée houle-courant. Un algorithme propre de calcul du coefficient de frottement au fond et d'autres paramètres cinématiques, pour l'action combinée houle-courant, a été proposé. Une vérification expérimentale de la méthode de calcul proposée a été effectuée. La possibilité de son application pour la détermination du début d'entraînement des sédiments a été mise en évidence.

Mots-clés : action combinée houle-courant, coefficient de frottement au fond, contrainte de cisaillement au fond, érosion, dynamique des sédiments, courant favorable

\section{Introduction}

Les travaux publiés, relatifs à l'étude de l'action combinée de la houle et des courants et son influence sur le mouvement des sédiments, notamment les travaux de recherche expérimentale en laboratoire et in situ, montrent que la superposition d'ondes superficielles sur la nappe d'écoulement peut provoquer un accroissement du débit solide $[1,2,3,4,5]$. Les données de ces travaux permettent 
de constater que, l'importance de l'influence de la houle sur le charriage des sédiments par le courant d'écoulement, est incontestable.

\section{$\underline{\text { 2. Estimation du coefficient de frottement }}$}

L'élaboration des modèles physiques convenables et de la méthode de calcul exige la détermination du coefficient de frottement au fond. Pour ce faire, deux approches ont été proposées $[6,7]$.

La première approche [6] donne une formulation du coefficient sommaire de frottement pour une interaction houle-courant favorable (écoulement dans le même sens que la propagation de la houle). Dans ce cas le coefficient de frottement est déterminé par la formule d'interpolation :

$$
f_{c w}=\frac{f_{w}+\alpha f_{c}}{1+\alpha}, \alpha=\frac{|U|}{U_{0}}
$$

Où : $\quad f_{w}$ - coefficient de frottement pour la houle seule

$\mathrm{f}_{\mathrm{c}}$ - coefficient de frottement le courant seul

$\mid \mathrm{U}$ - vitesse moyenne du courant

$\mathrm{U}_{0}$ - amplitude de la vitesse orbitale au fond

La formule (1) traduit les situations suivantes :

Dans le cas de l'action seule de la houle, $\alpha=0$ et $\mathrm{f}_{\mathrm{cw}}=\mathrm{f}_{\mathrm{w}}$. Par contre pour l'action seule du courant, $\alpha \rightarrow \infty$ et $\mathrm{fcw} \rightarrow \mathrm{f}_{\mathrm{c}}$. Il est à noter que cette simple approche a été proposée, vu le manque de données expérimentales et de modèles physiques pour l'étude du frottement au fond dans le cas de l'action combinée de la houle et du courant unidirectionnel [6].

Dans $[7,8]$, le coefficient de frottement au fond pour une interaction houlecourant, a été déterminé par la mesure expérimentale de la dissipation de la hauteur de houle le long du canal où le courant est horizontal homogène. L'inconvénient d'une telle approche est la difficulté d'obtention de précision suffisante des mesures de la dissipation de la hauteur de houle, qui, généralement est faible dans le canal. Les expériences $[7,8]$ ont montré que, comparativement au cas de l'action seule du courant, la superposition de la houle sur le courant entraîne une augmentation considérable du frottement du fond et par conséquent une diminution de la vitesse d'écoulement.

Un modèle physique permettant de déterminer le coefficient de frottement au fond pour une interaction houle-courant est proposé dans [11]. Les hypothèses de base de ce modèle sont les suivantes. :

A la superposition de la houle sur le courant, une interaction non linéaire des couches limites correspondantes se produit. Vu que la période de la houle est beaucoup plus petite que l'échelle spécifique du temps d'écoulement, la couche limite oscillatoire est considérée comme intégrée dans la couche limite du courant stationnaire. Si les vitesses orbitales maximales du fond sont de même ordre de grandeur que les vitesses du courant sur le fond, les contraintes de cisaillement sur le fond, générées par la houle, seront beaucoup plus importantes que celles du courant. Ceci est dû à la faiblesse de la couche limite oscillatoire. 
Dans la zone supérieure à la couche limite oscillatoire, le mélange turbulent se détermine uniquement par l'écoulement moyen. A l'intérieur de la couche limite oscillatoire, le mélange se détermine par l'action combinée de la houle et du courant. L'accroissement du mélange turbulent à l'intérieur de la couche limite oscillatoire entraîne la diminution des vitesses dans cette zone. Cet accroissement peut être décrit par l'introduction d'un coefficient fictif de rugosité $\mathrm{du}$ fond, dépassant la rugosité physique du fond. Le modèle physique, proposé dans [11], nécessite de futures vérifications expérimentales, mais présente l'intérêt d'être l'unique modèle physique de ce genre.

Le but du présent travail est la réalisation numérique du modèle [11], permettant d'obtenir la grandeur du coefficient de frottement du fond et autres caractéristiques du courant, ainsi que la vérification expérimentale de ce modèle. Pour la vérification expérimentale, nous avons utilisé les données [8], ainsi que les résultats de nos propres expériences.

\section{Algorithme de calcul}

Examinons l'algorithme de calcul des paramètres cinématiques du courant en présence de propagation d'ondes de surface. Pour la simplification du texte, nous appellerons courant combiné, le courant avec superposition d'ondes sur la surface libre.

Les données initiales de calcul sont :

$\left|\vec{U}_{c r}\right|$ - moyenne temporelle de vitesse du courant combiné mesurée au niveau $Z_{r}$ par rapport au fond ;

$\phi_{\mathrm{c}}$ - angle formé par les directions de la houle et du courant;

$\mathrm{U}_{0}$ - amplitude de la vitesse orbitale au fond;

$\mathrm{k}_{\mathrm{f}^{-}}$rugosité du fond.

L'algorithme peut être appliqué pour des orientations arbitraires des directions de la houle et du courant. Toutefois, il faut noter que, dans le cas de propagation de la houle dans le sens contraire au courant à grandes vitesses d'écoulement, il existe un effet non linéaire de déferlement et de blocage, exigeant une étude spécifique.

L'amplitude de la vitesse orbitale au fond se détermine par la formule déduite de la théorie de la houle :

$$
\left|\vec{U}_{0}\right|=\frac{\pi \mathrm{H}}{\operatorname{Tsh} 2 \pi \frac{\mathrm{d}}{\mathrm{L}}},
$$

Où : H- hauteur de la houle ;

d- profondeur du courant d'eau ;

$\mathrm{T}$ - période de la houle ;

L- longueur d'onde de la houle.

Dans ce cas, les éléments de la houle $(H, T, L)$ sont supposés connus : mesurés expériementalement ou déterminés par les modèles existants de développement ou de transformation de la houle par le courant. La rugosité du 
fond $\mathrm{k}_{\mathrm{f}}$ se détermine par le profil mesuré des vitesses du courant [7] ou se calcule par les relations connues pour les rugosités des grains ou des rides.

Les données initiales se présentent sous forme de quatre grandeurs adimensionnelles :

$$
\frac{\mathrm{z}_{\mathrm{r}}}{\mathrm{k}_{\mathrm{f}}} ; \quad \frac{\left|\overrightarrow{\mathrm{U}}_{\mathrm{cr}}\right|}{\left|\overrightarrow{\mathrm{U}}_{0}\right|} ; \quad \frac{\mathrm{k}_{\mathrm{f}}}{\left|\overrightarrow{\mathrm{A}}_{0}\right|} ; \phi_{\mathrm{c}},
$$

Où : $\left|\vec{A}_{0}\right|$ - amplitude orbitale de la houle.

$$
\left|\overrightarrow{\mathrm{A}}_{0}\right|=\frac{\left|\overrightarrow{\mathrm{U}}_{0}\right| \mathrm{T}}{2 \pi}
$$

En qualité d'approximation initiale pour la grandeur de vitesse du courant stationnaire à la hauteur a au dessus du fond, on prend :

$$
\frac{\left|\overrightarrow{\mathrm{U}}_{\mathrm{a}}\right|}{\left|\overrightarrow{\mathrm{U}}_{0}\right|}=\frac{\left|\overrightarrow{\mathrm{U}}_{\mathrm{cr}}\right|}{\left|\overrightarrow{\mathrm{U}}_{0}\right|} \text {. }
$$

Ici, il n'y a pas nécessité de concrétisation de la grandeur a

La valeur du coefficient de frottement au fond du courant combiné se détermine par la méthode itérative de résolution du modèle [11] :

$$
\begin{aligned}
& {\left[0,097\left(\frac{k_{f}}{A_{0}}\right)^{1 / 2} \frac{k}{f_{c w}^{3 / 4}}\right]^{2}+2\left[0,07\left(\frac{k_{f}}{\left|\vec{U}_{0}\right|}\right)^{1 / 2} \frac{k}{f_{c w}^{3 / 4}}\right]} \\
& {\left[\frac{V_{2}}{2 \alpha^{1 / 4}}\right] \cos \phi_{c}=\frac{\alpha^{3 / 4}}{4}-\frac{V_{2}^{2}}{4 \alpha^{1 / 2}},}
\end{aligned}
$$

Où : $\quad \mathrm{k}=\frac{1}{2 \xi_{0}^{1 / 2}} \frac{1}{\mathrm{~K}_{\mathrm{er}}^{2} 2 \xi_{0}^{1 / 2}+\mathrm{K}_{\mathrm{ei}}^{2} 2 \xi_{0}^{1 / 2}}$,

$$
\xi_{0}=\frac{2 \pi \mathrm{k}_{\mathrm{f}}}{30 \mathrm{Tk}\left|\overrightarrow{\mathrm{U}_{{ }_{\mathrm{cw}} \mathrm{w}}}\right|}
$$

Où : $\mathrm{K}_{\mathrm{er}}$ - fonction de Kelvin

k- constante de Karman.

$$
\left|\overrightarrow{\mathrm{U}^{*} \mathrm{cw}}\right|=\left(\frac{1}{2} \mathrm{f}_{\mathrm{cw}} \alpha\right)^{1 / 2}\left|\overrightarrow{\mathrm{U}}_{0}\right|,
$$

Où : $\quad \alpha=1+\left(\frac{\left|\overrightarrow{\mathrm{U}}_{\mathrm{a}}\right|}{\left|\overrightarrow{\mathrm{U}}_{0}\right|}\right)^{2}+2\left(\frac{\left|\overrightarrow{\mathrm{U}}_{\mathrm{a}}\right|}{\left|\overrightarrow{\mathrm{U}}_{0}\right|}\right) \cos \phi_{\mathrm{c}}$

$\mathrm{V}_{2}=\left(\frac{1}{2 \pi}\right)\left\{\left[\int_{-\theta^{*}}^{\pi+\theta^{*}}\left(g_{\mathrm{x}}^{4}+g_{\mathrm{y}}^{2} g_{\mathrm{x}}^{2}\right)^{1 / 2} d \theta-\int_{\pi+\theta^{*}}^{2 \pi-\theta^{*}}\left(g_{\mathrm{x}}^{4}+g_{\mathrm{x}}^{2} g_{\mathrm{y}}^{2}\right)^{1 / 2} d \theta\right]^{2}+\left[\int_{0}^{2 \pi}\left(g_{\mathrm{y}}^{4}+g_{\mathrm{x}}^{2} \mathrm{~g}_{\mathrm{y}}^{2}\right)^{1 / 2} d \theta\right]^{2}\right\}^{1 / 2}$ 
Où : $g_{x}=\left(\sin \theta+\frac{\left|\overrightarrow{\mathrm{U}}_{\mathrm{a}}\right|}{\left|\overrightarrow{\mathrm{U}}_{0}\right|} \cos \phi_{\mathrm{c}}\right)$;

$g_{\mathrm{y}}=\frac{\left|\overrightarrow{\mathrm{U}}_{\mathrm{a}}\right|}{\left|\overrightarrow{\mathrm{U}}_{0}\right|} \sin \phi_{\mathrm{c}}$

$\theta$ - angle de phase

$\theta_{*}=\sin ^{-1}\left(\frac{\left|\overrightarrow{\mathrm{U}}_{\mathrm{a}}\right|}{\left|\overrightarrow{\mathrm{U}}_{0}\right|} \cos \phi_{\mathrm{c}}\right)$

L'équation (14) admet une solution si $\frac{\left|\overrightarrow{\mathrm{U}}_{\mathrm{a}}\right|}{\left|\overrightarrow{\mathrm{U}}_{0}\right|} \leq \frac{1}{\cos \phi_{\mathrm{c}}}$.

Dans le cas où $\frac{\left|\overrightarrow{\mathrm{U}}_{\mathrm{a}}\right|}{\left|\overrightarrow{\mathrm{U}}_{0}\right|} \succ \frac{1}{\cos \phi_{\mathrm{c}}}$, on a : $\theta_{\star}=\frac{\pi}{2}$.

Ensuite examinons la nouvelle grandeur de l'angle formé par les directions de la houle et du courant, dans le cas de l'interaction houle-courant [11] :

$\bar{\phi}_{\mathrm{c}}=\operatorname{tg}^{-1}\left\{\left[\int_{0}^{2 \pi}\left(g_{\mathrm{y}}^{4}+\mathrm{g}_{\mathrm{x}}^{2} \mathrm{~g}_{\mathrm{y}}^{2}\right)^{1 / 2} \mathrm{~d} \theta\right]\left[\int_{-\theta_{*}}^{\pi+\theta_{*}}\left(g_{\mathrm{y}}^{4}+g_{\mathrm{x}}^{2} \mathrm{~g}_{\mathrm{y}}^{2}\right)^{1 / 2} \mathrm{~d} \theta-\int_{\pi+\theta_{*}}^{2 \pi-\theta_{*}}\left(g_{\mathrm{x}}^{4}+g_{\mathrm{x}}^{2} g_{\mathrm{y}}^{2}\right)^{1 / 2} \mathrm{~d} \theta\right]^{-1}\right\}$

La rugosité fictive du fond se calcule par la relation [11] :

$\frac{\left|\mathrm{k}_{\mathrm{fc}}\right|}{\left|\mathrm{k}_{\mathrm{f}}\right|}=\left[24 \frac{\left|\overrightarrow{\mathrm{U}{ }^{\mathrm{cw}}}\right|}{\left|\overrightarrow{\mathrm{U}}_{0}\right|} \mid \frac{\left|\overrightarrow{\mathrm{A}}_{0}\right|}{\left|\overrightarrow{\mathrm{k}}_{\mathrm{f}}\right|}\right]^{\beta}$

Où : $\quad \beta=\left(1-\frac{\left|\overrightarrow{\mathrm{U}_{{ }^{\mathrm{c}} \mathrm{c}}}\right|}{\left|\overrightarrow{\mathrm{U}_{0}}\right|} \frac{\left|\overrightarrow{\mathrm{U}}_{0}\right|}{\left|\overrightarrow{\mathrm{U}_{{ }^{\mathrm{c} \mathrm{CW}}}}\right|}\right)$.

Ici, la vitesse de frottement $\left|\overrightarrow{\mathrm{U}_{*_{\mathrm{c}}}}\right|$ est déterminée comme suit :

$$
\overrightarrow{\mid U_{*_{c}}}\left|=\left(\frac{1}{2} f_{c w} V_{2}\right)^{1 / 2}\right| \vec{U}_{0} \mid .
$$

La vérification de la convergence se fait par la relation :

$$
\left|\overrightarrow{\mathrm{U}}_{\mathrm{c}}\right|=\frac{\left|\overrightarrow{\mathrm{U}}_{\mathrm{c}}\right|}{\mathrm{k}} \ln \frac{30 \mathrm{z}}{\mathrm{k}_{\mathrm{fc}}}
$$


Le cycle se répète, tant que le rapport $\frac{\mathrm{k}_{\mathrm{fc}}}{\mathrm{k}_{\mathrm{f}}}$, déterminé par la relation (16), ne devienne inférieur au même rapport obtenu de la relation (19), ainsi :

$$
\left|\left(\frac{\mathrm{k}_{\mathrm{fc}}}{\mathrm{k}_{\mathrm{f}}}\right)_{16}-\left(\frac{\mathrm{k}_{\mathrm{fc}}}{\mathrm{k}_{\mathrm{f}}}\right)_{19}\right| \leq \varepsilon
$$

A la fin du calcul selon le cycle donné, on obtient l'ensemble de valeurs suivantes :

$$
\mathrm{F}_{\mathrm{cw}} ; \mathrm{k}_{\mathrm{fc}} ; \bar{\phi}_{\mathrm{c}} ;\left|\overrightarrow{\mathrm{U}_{*_{\mathrm{c}}}}\right| ;\left|\overrightarrow{\mathrm{U}_{{ }_{\mathrm{cw}}}}\right|
$$

L’algorithme et le programme ont été réalisés en langage Fortran.

\section{Résultats et discussion :}

D’abord utilisons les données des expériences [8], réalisées dans un canal $0,6 \times 0,75 \times 26 \mathrm{~m}$. Dans ces expériences, les caractéristiques cinématiques du courant ont été déterminées, pour une houle favorable (se propageant dans le même sens que le courant). Le coefficient de frottement au fond dans [8], a été calculé en mesurant la diminution de la hauteur de la houle, le long du canal. Le résultat essentiel de ces expériences est l'obtention de la diminution des vitesses du courant lors de la superposition de la houle, comparativement aux conditions similaires mais sans houle (fig.1). Dans [8], ce résultat s'explique par l'accroissement du frottement du fond dans le courant combiné, en comparaison avec le courant unidirectionnel. Toutefois, dans [8] on n'effectue pas de calculs quantitatifs correspondants.

Un calcul des caractéristiques cinématiques du courant, pour les conditions des expériences [8], présentées en figure 1, a été effectué selon l'algorithme décrit ci-dessus. Comme données initiales, on a utilisé les valeurs des vitesses du courant combiné, mesurées dans la zone proche du fond et représentées par des points sur la figure 1. La valeur de la rugosité physique prise dans ces expériences est : $\mathrm{k}_{0}=0,2 \mathrm{~cm}$ [8]. Dans les calculs par la formule (20), on a utilisé l'estimation de la moyenne quadratique des vitesses du courant. Les résultats de calcul des coefficients de frottement au fond, des vitesses moyennes du courant combiné et du courant initial unidirectionnel ainsi que les données des expériences et de calcul de [8] sont récapitulés dans le tableau ci-dessous : 


\begin{tabular}{|c|c|c|c|c|c|c|c|c|c|}
\hline \multirow[b]{3}{*}{ 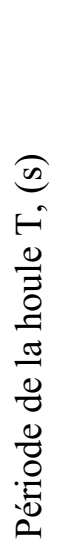 } & \multicolumn{4}{|c|}{ Courant de houle $\left(\mathrm{U}_{\mathrm{m}}=0\right)$} & \multicolumn{4}{|c|}{ Courant combiné $\left(\mathrm{U}_{\mathrm{m}}=0,374 \mathrm{~m} / \mathrm{s}\right)$} & \multirow[b]{3}{*}{ 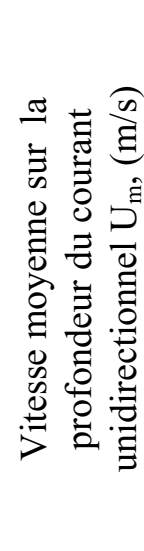 } \\
\hline & \multirow[b]{2}{*}{ 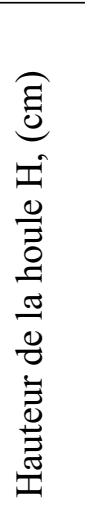 } & \multicolumn{3}{|c|}{$\begin{array}{l}\text { Coefficient de frottement } \\
\text { du fond } f_{w}\end{array}$} & \multirow[b]{2}{*}{ 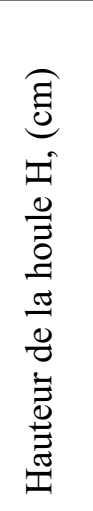 } & \multicolumn{3}{|c|}{$\begin{array}{l}\text { Coefficient de frottement } \\
\text { du fond } \mathrm{f}_{\mathrm{cw}}\end{array}$} & \\
\hline & & 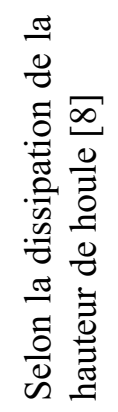 & $\begin{array}{l}\sigma \\
\sigma \\
\tilde{0} \\
0 \\
0 \\
0 \\
\tilde{0} \\
\overline{0} \\
\tilde{D}\end{array}$ & 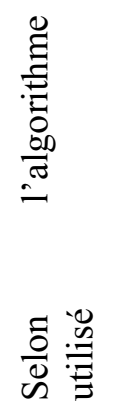 & & 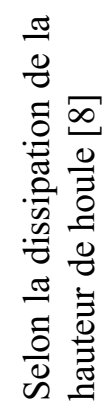 & $\begin{array}{l}\sigma \\
\sigma \\
0 \\
0 \\
0 \\
0 \\
0 \\
0 \\
0 \\
0\end{array}$ & 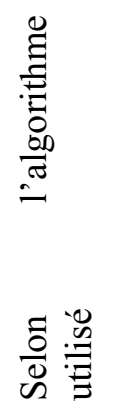 & \\
\hline \multirow{2}{*}{1,8} & 17,6 & 0,038 & 0,03 & 0,027 & 13,6 & 0,068 & 0,019 & 0,038 & 0,386 \\
\hline & 14,0 & 0,15 & 0,033 & 0,028 & 10,0 & 0,031 & 0,056 & 0,047 & 0,362 \\
\hline \multirow{2}{*}{1,5} & 17,8 & 0,033 & 0,034 & 0,030 & 12,0 & 0,030 & 0,016 & 0,048 & 0,372 \\
\hline & 14,4 & 0,085 & 0,038 & 0,033 & 9,6 & 0,011 & 0,032 & 0,055 & 0,348 \\
\hline
\end{tabular}

Les profils du courant moyen, calculés par la relation (19) sont représentés sur la figure 1. Les vitesses moyennes sur la profondeur du courant sont déterminées par intégration du profil (19). Il est à noter que, les profils théoriques logarithmiques du courant correspondent parfaitement à ceux mesurés dans la zone près du fond. Si l'on compare les profils mesurés sur toute la profondeur du courant, plus bas que le creux de la houle, on peut conclure que, les profils expérimentaux du courant combiné ont une forme plus lissée que ceux calculé par (19). Ceci est dû à l'hypothèse prise dans le modèle physique [11], qui considère qu'il n'y a pas d'influence de la houle sur le mélange turbulent dans la région située au dessus de la couche limite. La question de déformation du profil des vitesses moyennes du courant par la houle constitue en elle-même un thème particulier de recherche.

Dans la première moitié des colonnes du tableau, on a reporté les coefficients de frottement du fond pour un courant de houle. Ces coefficients ont été obtenus par expériences [8], par la formule de Jonson [6] et par calcul selon notre algorithme, pour une vitesse d'écoulement nulle $\left(\mathrm{U}_{\mathrm{m}}=0\right)$. La confrontation de ces résultats montre que, nos calculs vérifient, de manière entièrement satisfaisante, les résultats de calcul par la formule de Jonson. Les estimations [8] donnent un plus grand écart. Ceci est dû, à notre avis, au fait que, l'utilisation de la diminution de la hauteur de la houle dans un canal relativement court aboutit à une estimation grossière. L'importante dispersion des hauteurs mesurées de la houle dans $[7,8]$ confirme notre avis.

Dans la deuxième moitié des colonnes du tableau, ont été reportés les résultats de calcul pour les conditions de courant combiné et pour une vitesse moyenne de courant non agité $0,374 \mathrm{~m} / \mathrm{s}$. Les résultats de l'estimation du coefficients de frottement de fond $\mathrm{f}_{\mathrm{cw}}$, par la méthode de diminution de la hauteur de houle, par la formule de Jonson (1) et par notre calcul différent 
considérablement. L'estimation [8] conserve dans ce cas, le même inconvénient essentiel. En l'absence d'un quelconque critère raisonnable, on ne peut juger de la qualité de la formule (1). Pour la confirmation de nos calculs, nous avons donné dans la dernière colonne du tableau, les vitesses moyennes sur la profondeur du courant unidirectionnel, calculées pour chaque expérience. Ces dernières sont en concordance assez satisfaisante avec la valeur expérimentale $U_{m}=0,374 \mathrm{~m} / \mathrm{s}$, qui n'a pas été utilisée dans les calculs.

Pour la vérification expérimentale des méthodes de détermination du coefficient de frottement dans le courant combiné, examinons le problème de début d'entraînement des sédiments.

Il existe un nombre important de travaux de recherche sur la détermination du début d'entraînement des particules sédimentaires dans les courants unidirectionnels. La plupart de ces recherches présentent les résultats pour des conditions critiques sous forme de diagramme de Shields [12]. Le diagramme de Shields a été vérifié par un grand nombre d'expériences minutieuses menées par Knoroz [13], Ackers-White [10] et autres.

Plus tard, sur la base de nombreuses expériences réalisées par différents auteurs, il a été démontré [9] que, le diagramme de Shields peut être utilisé pour la détermination des conditions de début d'entraînement même dans le cas de courant de houle, si dans le calcul de la contrainte de cisaillement au fond qui rentre dans le paramètre de Shields, on utilise le coefficient de frottement de Jonson. Les résultats correspondants pour le courant unidirectionnel et le courant de houle, sont représentés dans [9] et montrés dans la figure 2.

Donc on peut dire que, pour le courant combiné, si le coefficient de frottement est assez bien déterminé, les conditions du début d'entraînement des sédiments doivent également correspondre au diagrammes de la figure 2, dans les limites de la dispersion des points expérimentaux présentés sur cette figure.

Cependant, les expériences sur le début d'entraînement des particules sédimentaires par le courant combiné, peuvent être utilisées pour l'estimation indirecte du coefficient de frottement au fond. Une telle approche est utilisée plus bas.

De tous les travaux qui nous sont connus, il existe un seul travail[5], dans lequel, selon les données de mesures in situ, on a déterminé les conditions du début d'entraînement des sédiments, sous l'action combinée de la houle et le courant. Les données de ce travail concernent le sable fin : $\mathrm{D}_{50}=0,003 \div 0,012 \mathrm{~cm}$ et $\mathrm{D}_{50}=0,012 \div 0,025 \mathrm{~cm}$.

En outre, des expériences ont été effectuées par nous, dans un canal de dimensions $0,8 \times 1,0 \times 13,6 \mathrm{~m}$ avec des sédiments de fractions plus grosses :

$\mathrm{D}_{50}=0,2 \mathrm{~cm} \mathrm{D}_{50}=0,1 \mathrm{~cm}$. Dans ces expériences on a généré une houle sur un courant favorable (dans le même sens que la propagation des ondes). Les conditions du début d'entraînement ont été déterminées selon le schéma de Knoroz[13].

Après avoir choisi le régime hydraulique du courant combiné, correspondant au début du mouvement des sédiments, nous avons effectué les mesures des hauteurs de la houle et des profils des vitesses sur la profondeur $\mathrm{du}$ courant dans trois sections distinctes le long du canal. En moyenne, les hauteurs mesurées de la houle diminuaient, toutefois la dispersion observée des valeurs est 
trop grande pour que cette information soit utilisée pour l'estimation du coefficient de frottement. C'est pourquoi, pour l'estimation du coefficient de frottement au fond, nous avons utilisé les valeurs mesurées de la vitesse du courant combiné selon la méthode décrite plus haut.

Les exemples de vitesses mesurées et calculées sont montrés dans la figure3. Tout comme dans les expériences [8], dans nos essais, nous avons observé, la diminution des vitesses moyennes du courant dans la zone proche du fond à la superposition de la houle. Nous avons également observé une certaine égalisation du profil de la vitesse moyenne sur la profondeur du courant. Ces effets étaient, ici, moins prononcés que dans les expériences $[7,8]$, ce qui se détermine par le rapport des échelles de la houle et du courant.

Nous avons utilisé les résultats de calcul de la vitesse de frottement $\left|\overrightarrow{\mathrm{U}_{* \mathrm{c}}}\right|$

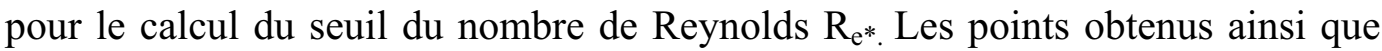
les résultats de traitement de ces points [5] sont présentés dans la figure 2. Ils concordent suffisamment avec le critère de Shields dans sa forme [9].

\section{Conclusion :}

Les résultats de notre travail nous permettent de conclure que, la méthode décrite ci-dessus, peut être utilisée pour le calcul du coefficient de frottement du fond $\mathrm{f}_{\mathrm{cw}}$ dans le cas de l'action combinée de la houle et du courant.
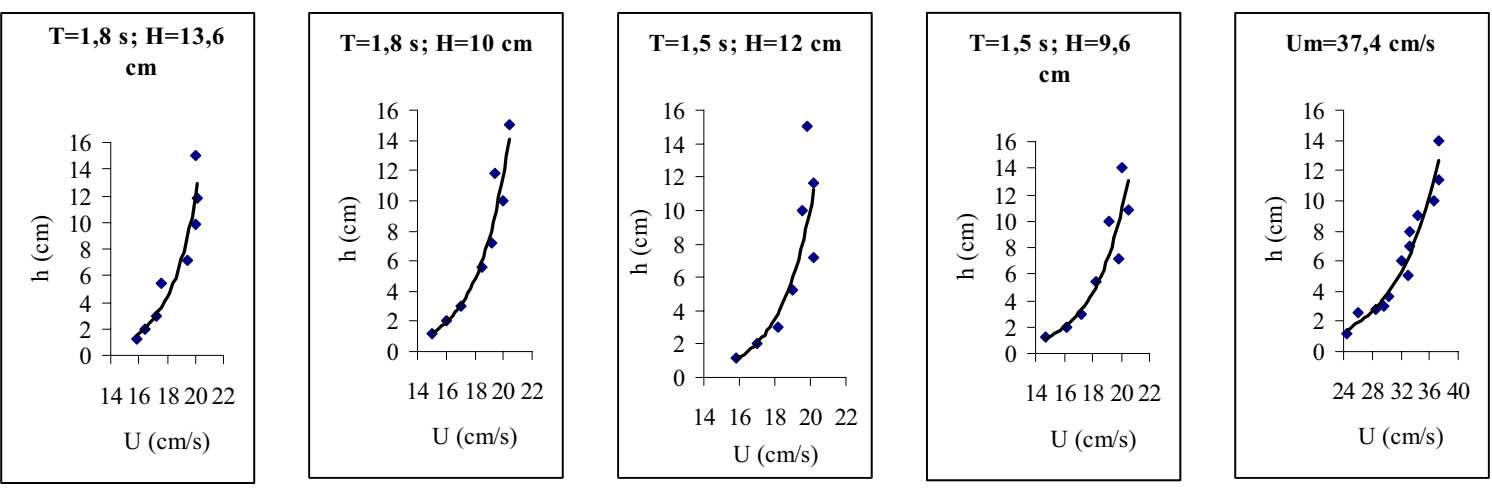

Figure 1 : Déformations du profil des vitesses moyennes du courant lors de superposition de la houle. La vitesse moyenne du courant unidirectionnel, initial est : Um=37,4 m/s [8] 


$$
\lg \operatorname{Re}_{*}=0,092\left(\lg D_{*}\right)^{2}+1,158 \lg D_{*}=0,367
$$

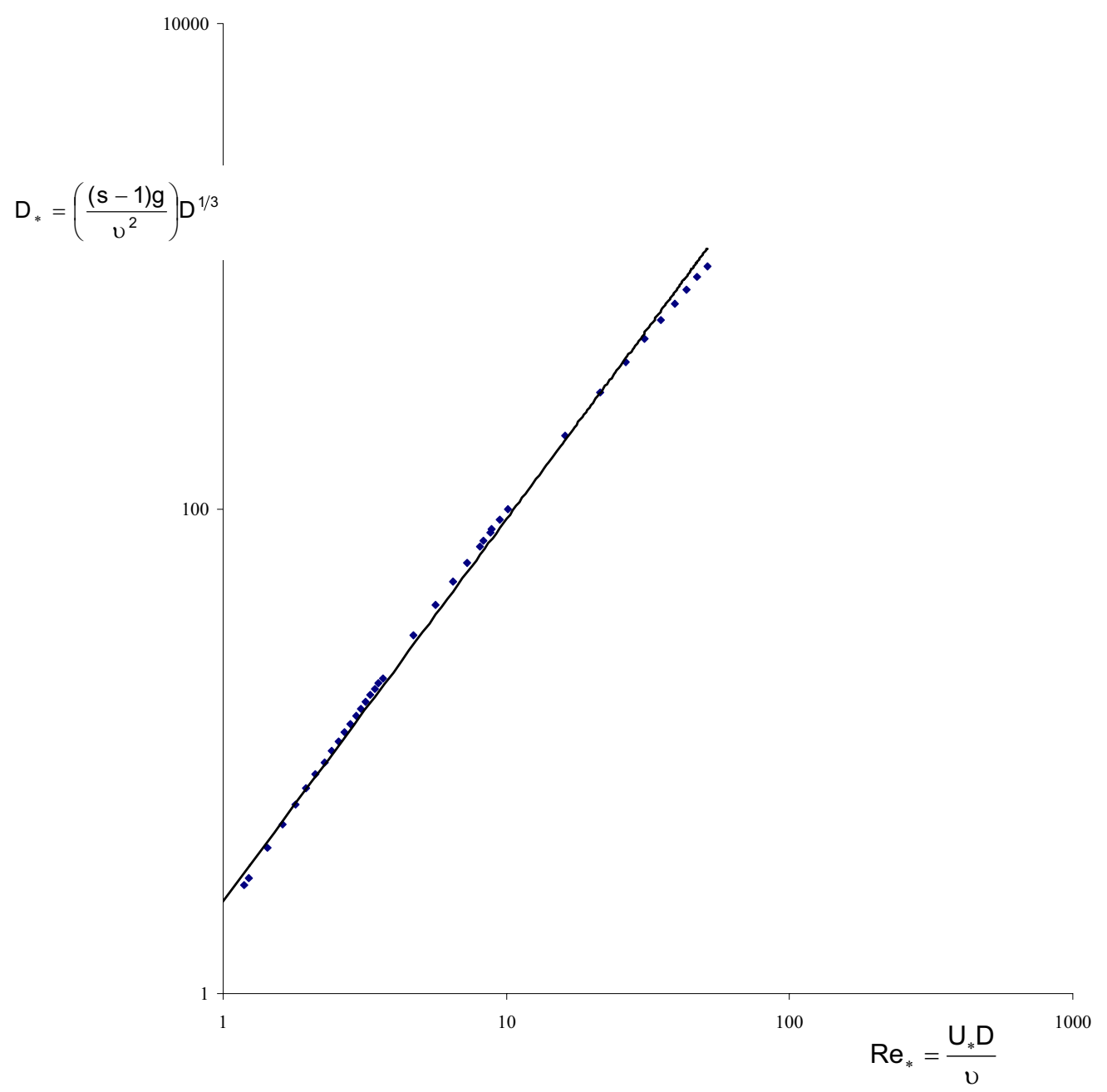

Figure 2 : Relation de Lenhoff [9] pour les conditions de début d'entraînement des sédiments.
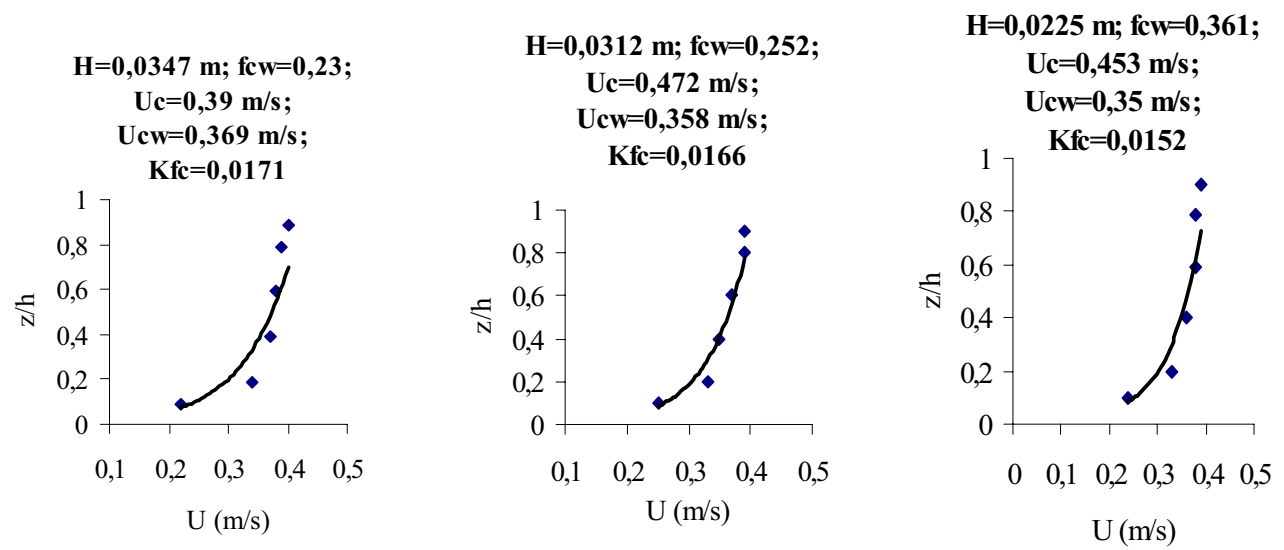

Figure 3 : Profil des vitesses du courant combiné dans les conditions de début d'entraînement des sédiments (pour $D_{50}=2 \mathrm{~mm}, T=0,67 \mathrm{~s} \& h=0,192 \mathrm{~m}$ ) 


\section{Références :}

1. Benoit C. \& Magnus L., Transport solide par charriage sous une interaction houle-courant, VIII ${ }^{\text {èmes }}$ journées nationales Génie civil-Génie côtier, Compiègne 7-8-9 septembre 2004,T.1, p.157-165

2. Catherine V., Modélisation du transport littoral, VIII ${ }^{\text {èmes }}$ journées nationales Génie civil-Génie côtier, Compiègne 7-8-9 septembre 2004, T.1, p.251-260

3. Marin F. \& Brossard J., Estimation des coefficients de frottement et de dissipation d'énergie dans la houle au voisinage d'un fond de rides, $V^{\text {èmes }}$ journées nationales Génie civil-Génie côtier, Toulon 13-14-15 mai 1998, p.27-34

4. Owen W., Thorh F.C., Effect of waves on sand transport by currents //Pras.16 ${ }^{\text {th }}$.Coast.Eng.conf., Hamburg, 1978, vol. 2.

5. Larsen L.H., Sternberg R.W., N.C., Marsden, M. Thomas, L. Field investigations of the threshold of grain motion by ocean waves and Currents., Mar. Geol 1981, 42: 165-182p.

6. Jonson J.G. The friction factor for a current superimposed by waves. Coastel. Eng.Lab. and Hydraul Lab., Techn. Univ. Denmark, Extract from Basic Res. Progr. Rep. No 11 , 1966, p. 2-12.

7. Brevik J., Bjorn Aas, Flume experiment on waves and currents ( I:Rippled Bed), Coast. Eng. N 3,1980, p. 149-177.

8. Brevik J. Flume experiment on waves and currents (Smooth bed). Coast. Eng. V. 4,1980, p. 89-110.

9. Lenhoff L. Incipient motion of particles under oscillatory flow. // Proc. 18 th. Coast. Eng. Conf, Cape Town, Nov. 14-19, 1982, V. 2, P. 1555-1562

10. Ackers P. White W.R., Sediment transport: New approach and analyse. Proceedings of the American Society of sivil Engineers. Vol. 99, Hyll November 1973, p. 2041-2060.

11. Grant W.D., Madsen O.S. Combined wove and current interaction with a rough bottom. Journal of geophysical research vol. 84, $\mathrm{N}^{\circ} \mathrm{CH}, 1979 \mathrm{p}$. 1797-1808

12. Shields A. Amvemdung der achulichters mechanic und der turbulenz forschung auf die geschiebewegund. Mitterlungen der preussischen Versuchsanstalt fur wosserbau und schiffbau. Heft 26, 1936.

13. Кнороз В.С. Неразмывающая скорость для несвязных грунтов и факторы её определяющие.- Изв.ВНИИГиМ. В.Е.Веденеева, 1958г, Т.5 Д, с.62-81. 